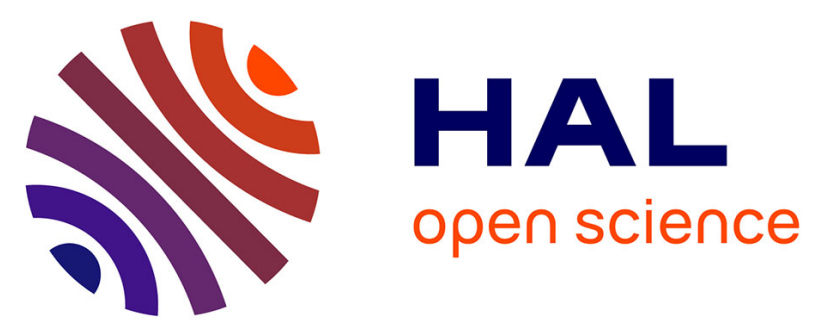

\title{
Direct and Regioselective C(sp3)-H Bond Fluorination of 2-Alkylazaarenes with Selectfluor
} Clothilde Le Guen, Ahmed Mazzah, Maël Penhoat, Patricia Melnyk, Christian Rolando, Laetitia Chausset-Boissarie

\section{- To cite this version:}

Clothilde Le Guen, Ahmed Mazzah, Maël Penhoat, Patricia Melnyk, Christian Rolando, et al.. Direct and Regioselective C(sp3)-H Bond Fluorination of 2-Alkylazaarenes with Selectfluor. Synthesis: Journal of Synthetic Organic Chemistry, 2020, 53 (6), pp.1157-1162. 10.1055/s-0040-1706482 . hal03061701

\section{HAL Id: hal-03061701 \\ https://hal.science/hal-03061701}

Submitted on 25 Nov 2021

HAL is a multi-disciplinary open access archive for the deposit and dissemination of scientific research documents, whether they are published or not. The documents may come from teaching and research institutions in France or abroad, or from public or private research centers.
L'archive ouverte pluridisciplinaire HAL, est destinée au dépôt et à la diffusion de documents scientifiques de niveau recherche, publiés ou non, émanant des établissements d'enseignement et de recherche français ou étrangers, des laboratoires publics ou privés. 


\section{Direct and regioselective $\mathrm{C}\left(\mathrm{sp}^{3}\right)-\mathrm{H}$ bond fluorination of 2-alkyl azaarenes with selectfluor}

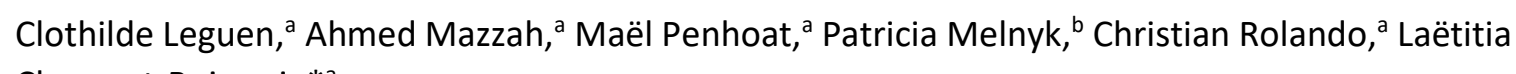
Chausset-Boissarie*a

aUniversity of Lille, USR CNRS 3290, (MSAP) Miniaturisation pour la Synthèse l'Analyse et la Protéomique, F-59000 Lille, France.

bUniversity of Lille, Inserm, CHU Lille, U1172-Lille Neuroscience \& Cognition, 59000 Lille, France.

laetitia.boissarie@univ-lille.fr

Abstract

Herein, a regioselective and direct mono-fluorination strategy of pyridylic and quinolinic $\mathrm{C}\left(\mathrm{sp}^{3}\right)-\mathrm{H}$ bonds was developed under transition metal free conditions with Selectfluor. The reaction was performed under smooth conditions and afforded fluorinated azaheterocycles including 2-methyl pyridines and quinolines in moderate to good yields.

Keywords : Fluorination, azaheterocycle, Selectfluor, 2-methylpyridines, 2-methylquinolines

\section{Introduction}

Nitrogen-containing aromatic heterocyles are abundant and ubiquitous scaffolds found in a large variety of natural products, agrochemicals, synthetic drugs and pharmaceuticals.

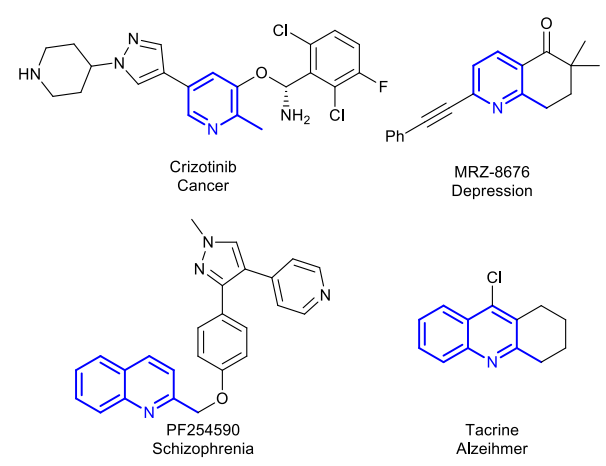

Figure 1. Selective examples of bioactive compounds bearing 2-alkyl substituted pyridine and quinoline core.

In particular, C2-substituted pyridines and quinolines exhibit a wide range of biological properties such as antimalarial, ${ }^{2}$ anticancer, ${ }^{3}$ anti-inflammatory ${ }^{4}$ or antidepressant activities ${ }^{5}$ as exemplified by compounds shown in Figure 1: Crizotinib, ${ }^{6}$ PF254590, ${ }^{7}$ MRZ-8676 ${ }^{8}$ and Tacrine $^{9}$ a well-known acetyl and butyrylcholinesterase inhibitor for the treatment of Alzheimer's disease withdrawn from its clinical use due to its liver toxicity. ${ }^{10}$ Additionally, they are versatile structural synthons in molecular synthesis. Since the introduction of fluorine atom into azaarenes hugely impacts their pharmacokinetic properties, prevents oxidative metabolism and more generally the overall biological activities of parent molecules, ${ }^{11}$ the development of novel methodologies for the late stage fluorination of 2-alkylpyridines and 2-alkylquinolines is still highly desirable. Numerous strategies were developed for the direct functionalization of a carbon adjacent to the quinoline or pyridine ring ${ }^{12}$ surprisingly, fluorination methods are limited. The classical synthetic approach for the monofluoration of alkylated pyridines and quinolines is based on fluorine displacement of a leaving group at the reaction site typically under harsh conditions (Figure 2, a). ${ }^{13}$ Recently, Shibatomi's group reported a decarboxylative fluorination of substituted 2-pyridylacetate to 2-(fluoroalkyl)pyridine, the main drawback of this method being the 
use of prefunctionalized substrates. ${ }^{14}$ During the last decade, significant progress has been made on the direct site selective fluorination of benzylic and aliphatic $\mathrm{C}\left(\mathrm{sp}^{3}\right)-\mathrm{H}$ bonds proceeding via transition metal catalysis, radical or photosensitized activation, ${ }^{15}$ however direct fluorination of pyridylic and quinolinic $\mathrm{C}\left(\mathrm{sp}^{3}\right)-\mathrm{H}$ bonds remains spare. In this context, efficient late-stage fluorination of 2alkylpyridine and 2-alkylquinoline under mild conditions is a particularly appealing and highly challenging task. Sandford and co-workers ${ }^{16}$ described the first pallado-catalyzed C-H fluorination of 8methylquinoline (Figure 2, b). Under this condition no fluorination was observed at the C2 positions of the quinoline ring. Pioneering work by the group of DesMarteau ${ }^{17}$ demonstrated that fluoromethylpyridines can be generated by electrophilic fluorination from 2- and 4-methyl pyridines in the presence of $\mathrm{N}$-fluoro-bis[(trifluoromethyl)sulfonyl]imide as fluorinated agent via an ionic mechanism. Since this seminal report, Britton and co-workers ${ }^{18}$ reported an elegant electrophilic mono-fluorination of 4-alkylpyridines including annulated pyridines and 4-alkylquinolines with $\mathrm{N}$-fluorobenzenesulfonimide (NFSI), however only a single example of fluorination of 2-annulated pyridine is described (Figure 2, c). Soon afterward, the group of Humbeck ${ }^{19}$ developed an efficient benzylic fluorination of 4-alkylquinolines through an unusual single-electron transfer thanks to the formation of a selectfluor/pyridine charge transfer complex (Figure 2, d). Nevertheless, only 4-alkylquinolines were employed and to the best of our knowledge no direct fluorination of 2-alkylquinolines or 2alkylpyridines have been described previously. Herein, we report a regioselective and direct fluorination on the $\mathrm{C} 2$ pyridylic or quinolinic carbon atoms under mild conditions.

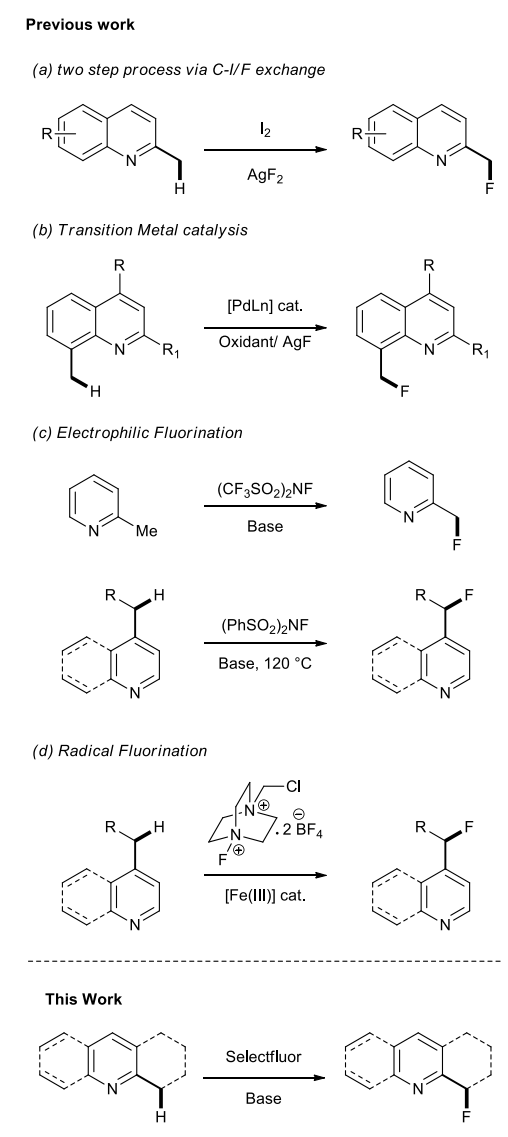

Figure 2. Direct fluorination of pyridylic and quinolinic $\mathrm{C}\left(\mathrm{sp}^{3}\right)-\mathrm{H}$ bonds.

\section{Results and discussion}

At the outset of the study, 2,3-cyclohexenopyridine (1a) was selected as model substrate to investigate the direct fluorination of 2-alkylazaarenes. Interestingly, fluorination with NFSI as fluorine source in acetonitrile under reflux, which is an effective system for direct fluorination of C4-alkylpyridines, ${ }^{18}$ was 
inefficient since no reaction was observed (Table 1, entry 1 ). To our pleasure, the use of one equivalent of selectfluor was found to be effective furnishing $2 \mathrm{a}$ after stirring in acetonitrile at $60{ }^{\circ} \mathrm{C}$ and inert atmosphere for $16 \mathrm{~h}$ albeit in low yield (Table 1, entry 2). Encouragingly, a reduced reaction temperature provided higher yield (Table 1, entry 3). Further optimization was then performed, starting from an investigation of the performance of difference bases. The reaction efficiency was further enhanced when performed with $\mathrm{NaHCO}_{3}$ (Table 1, entry 4) whereas poorer results were obtained with $\mathrm{Li}_{2} \mathrm{CO}_{3}$ or $\mathrm{Na}_{2} \mathrm{CO}_{3}$ (Table 1, entries 7-8). The addition of catalytic amount of AIBN as radical initiator or the iron(III) complex $\left[\mathrm{FeCl}_{4}\right]\left[\mathrm{FeCl}_{2}-(\mathrm{dmf})_{3}\right]^{19}$ did not further improve the fluorination process (Table 1, entries 5-6). The loading of the base was also tested and the use of 2 equivalent resulted in a slightly better yield (Table 1, entry 9). To our delight, we found that $62 \%$ of the desired product $2 \mathrm{a}$ could be obtained when both reactants are introduced simultaneity over $4 \mathrm{~h}$ to the reaction mixture (Table 1, entries 10).

Table 1 Optimization of the reaction conditions for the monofluoration of compound $1 \mathbf{a}^{\mathrm{a}}$

\begin{tabular}{|c|c|c|c|c|}
\hline Entry & Fluorine source & Base (x eq) & $\mathrm{T}\left({ }^{\circ} \mathrm{C}\right)$ & Yield $(\%)^{b}$ \\
\hline 1 & NFSI & - & 80 & Traces \\
\hline 2 & Selectfluor & - & 60 & 21 \\
\hline 3 & Selectfluor & - & 25 & 28 \\
\hline 4 & Selectfluor & $\mathrm{NaHCO}_{3}(1)$ & 25 & 37 \\
\hline $5^{c}$ & Selectfluor & $\mathrm{NaHCO}_{3}(1)$ & 25 & 25 \\
\hline $6^{d}$ & Selectfluor & $\mathrm{NaHCO}_{3}(1)$ & 25 & 22 \\
\hline 7 & Selectfluor & $\mathrm{Li}_{2} \mathrm{CO}_{3}(1)$ & 25 & 28 \\
\hline 8 & Selectfluor & $\mathrm{Na}_{2} \mathrm{CO}_{3}(1)$ & 25 & 21 \\
\hline 9 & Selectfluor & $\mathrm{NaHCO}_{3}(2)$ & 25 & 41 \\
\hline $10^{e}$ & Selectfluor & $\mathrm{NaHCO}_{3}(2)$ & 25 & 62 \\
\hline
\end{tabular}

aAll reactions unless specified were carried out using $1 \mathrm{a}(0.38 \mathrm{mmol}, 1 \mathrm{eq})$, Fluorine source (1 eq) and Base (1 eq), in $\mathrm{CH}_{3} \mathrm{CN}([0.25 \mathrm{M}])$ under $\mathrm{N}_{2}$ during $16 \mathrm{~h}$. ' Isolated yields. ' $10 \mathrm{~mol} \% 1,1^{\prime}$-azobis(cyclohexanecarbonitrile) as a radical initiator. d10 $\mathrm{mol}_{\%}\left[\mathrm{FeCl}_{4}\right]\left[\mathrm{FeCl}_{2}\right.$-(dmf) 3 as catalyst. eco-addition of solutions of Selectfluor and 1a over $4 \mathrm{~h}$.

The generality and limitations of the direct fluorination of 2-alkylazaarenes were then investigated (scheme 1). Firstly, various pyridine derivatives were employed to react with selectfluor with yields ranging from 18 to $62 \%$ (scheme 1, 2a-f). We were pleased to find that 6,7-dihydro-5Hcyclopenta[b]pyridine afforded the monofluorinated product $\mathbf{2} \mathbf{b}$ in decent yield. When very challenging 2-methylpyridine was employed as substrate the reaction could proceed to provide the desired product $\mathbf{2 c}$ albeit in low yield but within the same yield range than the procedure using the highly reactive fluorinating agent $\left(\mathrm{CF}_{3} \mathrm{SO}_{2}\right)_{2} \mathrm{NF}$. It was observed that 2-fluoro-ethylpyridine $\mathbf{2 d}$ was obtained with lower yield compared to 2-fluoro-methylpyridine however when the benzylic position is stabilized fluorinated adduct $\mathbf{2 e}$ could be obtained with good yield. Interestingly, fluorination of 2,4dimethylpyridine provided 4-methyl-2-fluoromethylpyridine $\mathbf{2} \mathbf{d}$ with a complete regioselectivity. Thus, this methodology could represent an interesting opportunity for a late stage site selective fluorination in case of another benzylic position was present in the molecule. To further explore the scope of the reaction, various quinolines were employed. 2-methylquinolines proved to be reactive toward selectfluor with a slight modification of the reaction conditions providing the corresponding fluorinated products in low to good yields (18-48\%). 2-methylquinolines bearing electron donating group (-Me, $-\mathrm{Ph}) \mathbf{1} \mathbf{h}$ and $\mathbf{1 i}$ on the phenyl ring gave the desired product in higher yields compared to those with electro-withdrawing substituents $(-\mathrm{Br},-\mathrm{Cl}) \mathbf{1} \mathbf{j}$ and $\mathbf{1} \mathbf{k}$. Unfortunately, strong electro- 
withdrawing group $\left(\mathrm{NO}_{2}\right)$ containing 2-methylquinoline 1e was not tolerated under the reaction conditions. Though 2-methylquinolines deliver fluorinated compounds with low to moderate yields this represent the first example of direct fluorination of 2-methylquinoline derivatives. As for pyridines, the reaction also proceed with complete regioselectivity to give $2 \mathrm{~m}$ in $32 \%$ yield. The fluorination was also compatible with 1,2,3,4-tetrahydroacridines $\mathbf{1 n}$ and 10, decent yield could be obtained and halogen bearing substrate 10 could offer an opportunity to further functionalize the product. In any case, the formation of by-products was not observed and the unreacted remaining 2-alkylazaarenes can be recoverable.

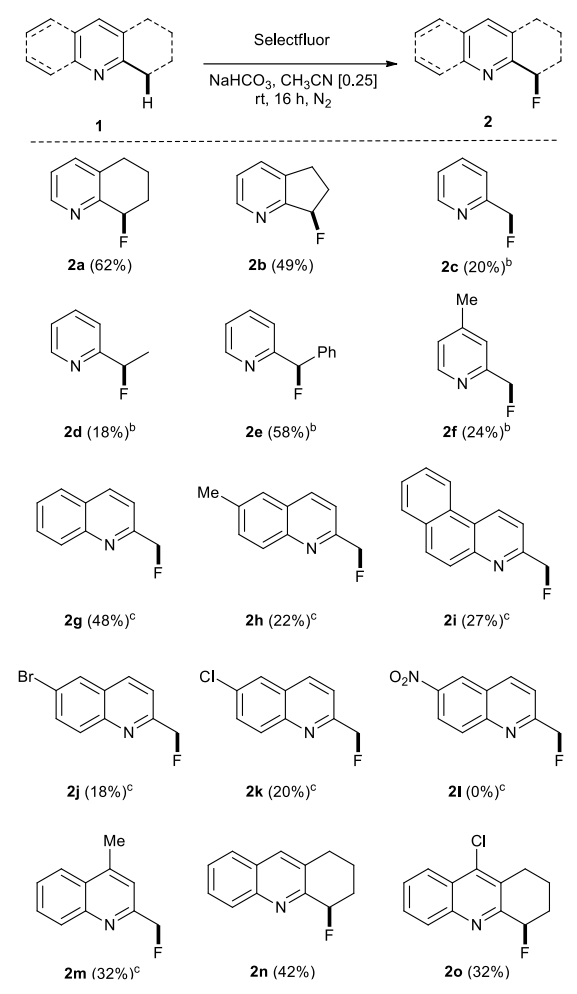

Scheme 1. ${ }^{a}$ All reactions unless specified were carried out using $1 \mathrm{a}(0.38 \mathrm{mmol}, 1 \mathrm{eq})$, Fluorine source $(1 \mathrm{eq})$ and Base (2 eq), in $\mathrm{CH}_{3} \mathrm{CN}$ ([0.25 M] under $\mathrm{N}_{2}$ during $16 \mathrm{~h}$. ${ }^{b} \mathrm{NMR}$ yields. ${ }^{\mathrm{c}} 1 \mathrm{a}(0.38 \mathrm{mmol}, 1 \mathrm{eq})$, Fluorine source (2 eq) and Base (3 eq), in $\mathrm{CH}_{3} \mathrm{CN}([0.25 \mathrm{M}])$ at $35^{\circ} \mathrm{C}$ under $\mathrm{N}_{2}$ during $16 \mathrm{~h}$.

To gain insights into the fluorination mechanism, we conducted some control experiment under the standard conditions. 2-alkylazaarenes usually serve as nucleophilic coupling partner toward fluorinated agents such as NFSI. ${ }^{18}$ Ended, 2-azaheterocycles can be first converted to the corresponding $\mathrm{N}$-fluoropyridinium salt via addition to the fluorinating agent. Then, after the loss of one proton on the methylene site the corresponding tautomer can react with selectfluor to provide the pyridylic C-F bond. Finally, 2-alkylpyridines can attack the resulting pyridinium to deliver fluoroalkylazaarenes and the pyridinium intermediate. ${ }^{19}$ However, the reaction did not proceed with $\mathrm{N}$-fluoropyridinium triflate as fluorine source. These results suggested that a radical pathway rather than an ionic pathway ${ }^{16}$ might be involved. As also described by Humbeck ${ }^{19}$ we evaluated the formation of pyridine/Selectfluor charge-transfer complex in solution using UV/Vis spectroscopy. The 2,3-cyclohexenopyridine solution showed a maximum absorbance at a wavelength of $269 \mathrm{~nm}$, however the absorbance of the reaction mixture under identical concentration was more intense, with its wavelength nearly identical to the 2,3-cyclohexenopyridine maximum.

On the basis of these investigations and literature precedents for benzylic fluorination of C4-alkylated $\mathrm{N}$-heterocycles with selectfluor ${ }^{17}$ a plausible mechanism is given in scheme 3 . At first, a charge-transfer complex between alkylquinolines or alkylpyridines and selectfluor is formed. Then, the complex 
exhibits a stepwise single electron transfer or a concerted proton-coupled electron transfer to generate the $\mathrm{C} 2$ pyridylic or quinolinic radical which undergoes a fluorine atom transfer to yield the desired fluorinated compound.

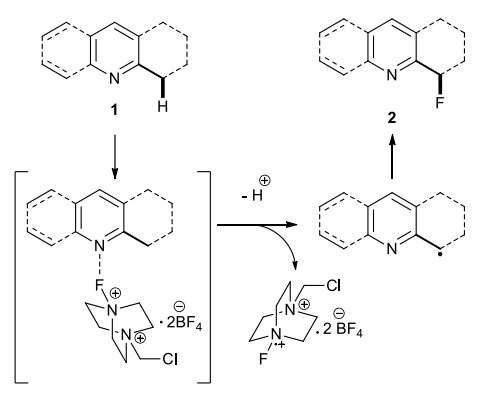

Scheme 2. Mechanistic proposal for the fluorination of pyridylic and quinolinic $\mathrm{C}\left(\mathrm{sp}^{3}\right)-\mathrm{H}$ bonds.

In conclusion, we successfully developed the direct and regioselective fluorination of the $\mathrm{C}\left(\mathrm{sp}^{3}\right)-\mathrm{H}$ bonds of 2-alkylpyridine and 2-alkylquinoline derivatives under mild condition with selectfluor. Various fluorinated $\mathrm{N}$-heterocycles were synthesized in low to good yields. The mechanistic study suggested that the fluorination might proceed via a radical pathway. This work provides a useful tool for the synthesis of $\mathrm{C} 2$-fluoromethylazaarenes which are versatile skeletons for medicinal chemistry.

\section{Experimental section}

All reagents were purchased from commercial suppliers (Strem Chemicals Inc., Sigma-Aldrich or Alfa Aesar) and were used without further purification unless otherwise indicated. Thin-layer chromatography plates (Silica gel 60 F254, Merck) and visualized under UV ( $254 \mathrm{~nm}$ ). Chromatographic purifications were performed using PuriFlash ${ }^{\circledR} 215$ equipped with UV (90-840 nm) and ELSD detectors purchased from Interchim. Pre-packed flash chromatography Silica HP columns $(30 \mu \mathrm{m})$ were used. ${ }^{1} \mathrm{H}$ and ${ }^{13} \mathrm{C}$ were recorded on a Bruker AVANCE 300 spectrometer at $300 \mathrm{MHz}(75 \mathrm{MHz})$. High-resolution mass spectra (HRMS) were performed on a Thermo LTQ orbitrap mass spectrometer.

Procedures

General procedure A

To an oven-dried flask, $\mathrm{NaHCO}_{3}$ was added $(0.750 \mathrm{mmol}, 2$ equiv.) followed by a co-addition over $4 \mathrm{~h}$ of a solution of nitrogen heterocycles $(0.375 \mathrm{mmol}, 1$ equiv.) and a solution of selectfluor $(0.375 \mathrm{mmol}$, 1 equiv.) in dry acetonitrile $(1.5 \mathrm{~mL},[0.25])$. The reaction mixture was then stirred for $16 \mathrm{~h}$ at room temperature gradually forming an orange brown solution. The reaction mixture was diluted with dichloromethane, washed with a saturated $\mathrm{NaHCO}_{3}$ solution and extracted with dichloromethane. The combined organic layers were dried with $\mathrm{MgSO}_{4}$, filtered and concentrated in vacuo. The residue was purified by flash column chromatography on silica gel (Petroleum ether/EtOAc $=100: 0$ to $95: 5$ ) to give fluorinated nitrogen heterocycles.

\section{General procedure B}

To an oven-dried flask, to a solution of nitrogen heterocycles $(0.375 \mathrm{mmol}, 1$ equiv. $)$ in dry acetonitrile ( $3.0 \mathrm{~mL}$, [0.12]) was added $\mathrm{NaHCO}_{3}(1.12 \mathrm{mmol}, 3$ equiv.) and selectfluor ( $0.75 \mathrm{mmol}, 2$ equiv.). The reaction mixture was then stirred for $16 \mathrm{~h}$ at $35{ }^{\circ} \mathrm{C}$ gradually forming an orange brown solution. The reaction mixture was diluted with dichloromethane, washed with a saturated $\mathrm{NaHCO}_{3}$ solution and extracted with dichloromethane. The combined organic layers were dried with $\mathrm{MgSO}_{4}$, filtered and concentrated in vacuo. The residue was purified by flash column chromatography on silica gel (Petroleum ether/EtOAc $=100: 0$ to 90:10) to give fluorinated nitrogen heterocycles. 


\section{8-Fluoro-5,6,7,8-tetrahydroquinoline (2a)}

Procedure A; Yellow oil, yield: $35.1 \mathrm{mg}(62 \%)$.

${ }^{1} \mathrm{H}$ NMR $\left(\mathrm{CDCl}_{3}, 300 \mathrm{MHz}\right): \delta=8.54(\mathrm{dd}, \mathrm{J}=4.6,0.7 \mathrm{~Hz}, 1 \mathrm{H}), 7.48(\mathrm{~d}, \mathrm{~J}=7,8 \mathrm{~Hz}, 1 \mathrm{H}),$,7.21 (ddd, J = 7.7, 4.7, $2.0 \mathrm{~Hz}, 1 \mathrm{H}), 5,56(\mathrm{dt}, \mathrm{J}=49.8,3.3 \mathrm{~Hz}, 1 \mathrm{H}), 2.90-2.69(\mathrm{~m}, 2 \mathrm{H}), 2.4-2.32(\mathrm{~m}, 2 \mathrm{H}), 2.06-1.81(\mathrm{~m}, 3 \mathrm{H})$. ${ }^{13} \mathrm{C} \mathrm{NMR}\left(\mathrm{CDCl}_{3}, 300 \mathrm{MHz}\right): \delta=152.4,148.2$ (d, J = 2.5 Hz), 137.4, 133.8, 124.0 (d, J = 3.5 Hz), 88.9 (d, J $=168.0 \mathrm{~Hz}), 29.8(\mathrm{~d}, \mathrm{~J}=21.4 \mathrm{~Hz}), 28.4,17.6$.

${ }^{19} \mathrm{~F} \mathrm{NMR}\left(\mathrm{CDCl}_{3}, 282.2 \mathrm{MHz}\right): \delta=-163.0-163.3(\mathrm{~m}, 1 \mathrm{~F})$.

HRMS: $\mathrm{m} / \mathrm{z}[\mathrm{M}+\mathrm{H}]+$ calc. for $\mathrm{C}_{9} \mathrm{H}_{11} \mathrm{FN}, 152.0870$ found 152.0868 .

\section{7-Fluoro-6,7-dihydro-5H-cyclopenta[b]pyridine (2b) ${ }^{18 a}$}

Procedure A; Yellow oil, yield: $25.2 \mathrm{mg}(49 \%)$.

${ }^{1} \mathrm{H} \mathrm{NMR}\left(\mathrm{CDCl}_{3}, 300 \mathrm{MHz}\right): \delta=8.53(\mathrm{~d}, \mathrm{~J}=4.6 \mathrm{~Hz}, 1 \mathrm{H}), 7.62(\mathrm{~d}, \mathrm{~J}=7.7 \mathrm{~Hz}, 1 \mathrm{H}), 5.91$ (ddd, J = 56.5, 6.3, $2.8 \mathrm{~Hz}, 1 \mathrm{H}), 3.15(\mathrm{ddd}, \mathrm{J}=10.8,8.0,3.4 \mathrm{~Hz}, 1 \mathrm{H}), 3.01-2.74(\mathrm{~m}, 1 \mathrm{H}), 2.64-2.19(\mathrm{~m}, 2 \mathrm{H})$.

${ }^{13} \mathrm{C} \mathrm{NMR}\left(\mathrm{CDCl}_{3}, 300 \mathrm{MHz}\right): \delta=160.0(\mathrm{~d}, \mathrm{~J}=15.8 \mathrm{~Hz}), 149.1,138.2(\mathrm{~d}, \mathrm{~J}=4.0 \mathrm{~Hz}), 133.5,124.3,95.7$ (d, $\mathrm{J}=175.2 \mathrm{~Hz}), 30.8(\mathrm{~d}, \mathrm{~J}=22.7 \mathrm{~Hz}), 27.9$.

${ }^{19} \mathrm{~F}$ NMR $\left(\mathrm{CDCl}_{3}, 282.2 \mathrm{MHz}\right): \delta=167.7(\mathrm{dt}, \mathrm{J}=56.5,28.0 \mathrm{~Hz})$.

HRMS: $\mathrm{m} / \mathrm{z}[\mathrm{M}+\mathrm{H}]+$ calc. for $\mathrm{C}_{8} \mathrm{H}_{9} \mathrm{FN}, 131.0165$ found 131.0163 .

$\underline{\text { 2-(Fluoromethyl)pyridine }(2 \mathrm{c})^{17}}$

Procedure A; Yellow oil, Yield determined by analysis of ${ }^{1} \mathrm{H}-\mathrm{NMR}$ spectra of crude reaction product using 4-fluorotoluene as internal standard: $20 \%$ yield.

${ }^{1} \mathrm{H} \mathrm{NMR}\left(\mathrm{CDCl}_{3}, 300 \mathrm{MHz}\right): \delta=8.57(\mathrm{~d}, \mathrm{~J}=4.8 \mathrm{~Hz}, 1 \mathrm{H}), 7.74(\mathrm{td}, \mathrm{J}=7.7,1.7 \mathrm{~Hz}, 1 \mathrm{H}), 7.45(\mathrm{~d}, \mathrm{~J}=7.9 \mathrm{~Hz}$, $1 \mathrm{H}), 7.26-7.19(\mathrm{~m}, 1 \mathrm{H}), 5.48(\mathrm{~d}, \mathrm{~J}=46.9 \mathrm{~Hz}, 2 \mathrm{H})$.

${ }^{13} \mathrm{C} \mathrm{NMR}\left(\mathrm{CDCl}_{3}, 300 \mathrm{MHz}\right): \delta=156.5(\mathrm{~d}, \mathrm{~J}=21.2 \mathrm{~Hz}), 149.3,137.1,123.2,120.7(\mathrm{~d}, \mathrm{~J}=5.7 \mathrm{~Hz}), 84.5(\mathrm{~d}$, $\mathrm{J}=169.6 \mathrm{~Hz})$.

${ }^{19} \mathrm{~F} \mathrm{NMR}\left(\mathrm{CDCl}_{3}, 282.2 \mathrm{MHz}\right): \delta=-221.1(\mathrm{t}, \mathrm{J}=46.9 \mathrm{~Hz})$.

HRMS: $\mathrm{m} / \mathrm{z}[\mathrm{M}+\mathrm{H}]+$ calc. for $\mathrm{C}_{6} \mathrm{H}_{7} \mathrm{FN}, 112.0557$ found 112.0555 .

\section{2-(1-Fluoroethyl)pyridine (2d)}

Procedure A; Yield determined by analysis of ${ }^{1} \mathrm{H}-\mathrm{NMR}$ spectra of crude reaction product using 4fluorotoluene as internal standard: $16 \%$ yield.

${ }^{1} \mathrm{H} \mathrm{NMR}\left(\mathrm{CDCl}_{3}, 300 \mathrm{MHz}\right): \delta=8.64-8.63(\mathrm{~m}, 2 \mathrm{H}), 7.28(\mathrm{~d}, 1 \mathrm{H}, \mathrm{J}=6.0 \mathrm{~Hz}), 5,63(\mathrm{dt}, 1 \mathrm{H}, \mathrm{J}=47.4,6.6 \mathrm{~Hz})$, 1.64 (dd, $3 \mathrm{H}, \mathrm{J}=24.3,6.6 \mathrm{~Hz}$ ).

${ }^{13} \mathrm{C} \mathrm{NMR}\left(\mathrm{CDCl}_{3}, 300 \mathrm{MHz}\right): \delta=152,3(\mathrm{~d}, \mathrm{~J}=16.0 \mathrm{~Hz}), 147.9(\mathrm{~d}, \mathrm{~J}=2.5 \mathrm{~Hz}), 137.2,133.6,128.8(\mathrm{~d}, \mathrm{~J}=$ $3.6 \mathrm{~Hz}), 88.8(\mathrm{~d}, \mathrm{~J}=168.0 \mathrm{~Hz}), 29.6(\mathrm{~d}, \mathrm{~J}=21.8 \mathrm{~Hz}), 28.2,17.4(\mathrm{~d}, \mathrm{~J}=2.0 \mathrm{~Hz})$.

${ }^{19} \mathrm{~F} \mathrm{NMR}\left(\mathrm{CDCl}_{3}, 282.2 \mathrm{MHz}\right): \delta=-176.9(\mathrm{dq}, 1 \mathrm{~F}, \mathrm{~J}=24.3,47.4 \mathrm{~Hz})$.

HRMS: $\mathrm{m} / \mathrm{z}[\mathrm{M}+\mathrm{H}]+$ calc. for $\mathrm{C}_{7} \mathrm{H}_{9} \mathrm{FN}, 126.0714$ found 126.0712 .

2-(Fluoro(phenyl)methyl)pyridine (2e)

Procedure A; Yellow oil, yield: $40.6 \mathrm{mg}$ (58\%).

${ }^{1} \mathrm{H} \mathrm{NMR}\left(\mathrm{CDCl}_{3}, 300 \mathrm{MHz}\right): \delta=8.38(\mathrm{~d}, \mathrm{~J}=4.8 \mathrm{~Hz}, 1 \mathrm{H}), 7.50(\mathrm{t}, \mathrm{J}=7.7 \mathrm{~Hz}, 1 \mathrm{H}), 7.36(\mathrm{~d}, \mathrm{~J}=7.8 \mathrm{~Hz}, 1 \mathrm{H})$, $7.33-7.11(\mathrm{~m}, 5 \mathrm{H}), 6.97(\mathrm{dd}, \mathrm{J}=17.2,9.9 \mathrm{~Hz}, 1 \mathrm{H}), 6.38(\mathrm{~d}, \mathrm{~J}=46.7 \mathrm{~Hz}, 1 \mathrm{H})$.

${ }^{13} \mathrm{C} \mathrm{NMR}\left(\mathrm{CDCl}_{3}, 300 \mathrm{MHz}\right): \delta=159.1(\mathrm{~d}, \mathrm{~J}=26.6 \mathrm{~Hz}), 149.1(\mathrm{~d}, \mathrm{~J}=2.2 \mathrm{~Hz}), 138.8(\mathrm{~d}, \mathrm{~J}=21.1 \mathrm{~Hz}), 136.9$, $128.7(\mathrm{~d}, \mathrm{~J}=2.2 \mathrm{~Hz}), 128.5,126.6(\mathrm{~d}, \mathrm{~J}=6.5 \mathrm{~Hz}), 123.0,120.0(\mathrm{~d}, \mathrm{~J}=5.6 \mathrm{~Hz}), 94.7(\mathrm{~d}, \mathrm{~J}=174.2 \mathrm{~Hz})$.

${ }^{19} \mathrm{~F} \mathrm{NMR}\left(\mathrm{CDCl}_{3}, 282.2 \mathrm{MHz}\right): \delta=-174.62(\mathrm{~d}, \mathrm{~J}=46.7 \mathrm{~Hz})$.

HRMS: $\mathrm{m} / \mathrm{z}[\mathrm{M}+\mathrm{H}]+$ calc. for $\mathrm{C}_{12} \mathrm{H}_{11} \mathrm{FN}, 188.0870$ found 188.0873 .

$\underline{\text { 2-(Fluoromethyl)-4-methylpyridine (2f) }}$ 
Procedure A; Yield determined by analysis of $1 \mathrm{H}-\mathrm{NMR}$ spectra of crude reaction product using 4fluorotoluene as internal standard: $24 \%$ yield.

${ }^{1} \mathrm{H} \mathrm{NMR}\left(\mathrm{CDCl}_{3}, 300 \mathrm{MHz}\right): \delta=8.41(\mathrm{~d}, \mathrm{~J}=5.0 \mathrm{~Hz}, 1 \mathrm{H}), 7.34-7.20(\mathrm{~m}, 1 \mathrm{H}), 7.13-6.93(\mathrm{~m}, 1 \mathrm{H}), 5.44(\mathrm{~d}$, $\mathrm{J}=47.0 \mathrm{~Hz}, 2 \mathrm{H}), 2.37(\mathrm{~s}, 3 \mathrm{H})$.

${ }^{13} \mathrm{C} \mathrm{NMR}\left(\mathrm{CDCl}_{3}, 300 \mathrm{MHz}\right): \delta=156.2(\mathrm{~d}, \mathrm{~J}=21.0 \mathrm{~Hz}), 149.1,148.3,124.1,121.6$ (d, J = 5.5 Hz), 84.6 (d, $\mathrm{J}=169.4 \mathrm{~Hz}), 21.2$.

${ }^{19} \mathrm{~F} \mathrm{NMR}\left(\mathrm{CDCl}_{3}, 282.2 \mathrm{MHz}\right): \delta=-220.8(\mathrm{t}, \mathrm{J}=47.0 \mathrm{~Hz})$.

HRMS: $\mathrm{m} / \mathrm{z}[\mathrm{M}+\mathrm{H}]+$ calc. for $\mathrm{C}_{7} \mathrm{H}_{9} \mathrm{FN}, 126.0714$ found 126.0715 .

$\underline{\text { 2-(Fluoromethyl)quinoline }(\mathbf{2 g})^{13}}$

Procedure B; Brown solid, yield: $29.0 \mathrm{mg}(46 \%)$.

${ }^{1} \mathrm{H} \mathrm{NMR}\left(\mathrm{CDCl}_{3}, 300 \mathrm{MHz}\right): \delta=8.11(\mathrm{~d}, \mathrm{~J}=8.5 \mathrm{~Hz}, 1 \mathrm{H}), 7.96(\mathrm{~d}, \mathrm{~J}=8.5 \mathrm{~Hz}, 1 \mathrm{H}), 7.72(\mathrm{~d}, \mathrm{~J}=8.1 \mathrm{~Hz}, 1 \mathrm{H})$, 7.63 (ddd, J = 8.5, 6.9, $1.5 \mathrm{~Hz}, 1 \mathrm{H}), 7.53-7.39(\mathrm{~m}, 2 \mathrm{H}), 5.56(\mathrm{~d}, \mathrm{~J}=47.0 \mathrm{~Hz}, 2 \mathrm{H}$ ).

${ }^{13} \mathrm{C} \mathrm{NMR}\left(\mathrm{CDCl}_{3}, 300 \mathrm{MHz}\right): \delta=156.9(\mathrm{~d}, \mathrm{~J}=21.8 \mathrm{~Hz}), 147.5,137.2,130.0,129.1,127.8,127.8,126.8$, $118.3(\mathrm{~d}, \mathrm{~J}=5.1 \mathrm{~Hz}), 85.1(\mathrm{~d}, \mathrm{~J}=170.3 \mathrm{~Hz})$.

${ }^{19} \mathrm{~F} \mathrm{NMR}\left(\mathrm{CDCl}_{3}, 282.2 \mathrm{MHz}\right): \delta=-221.30(\mathrm{t}, \mathrm{J}=47.0 \mathrm{~Hz})$.

HRMS: $\mathrm{m} / \mathrm{z}[\mathrm{M}+\mathrm{H}]+$ calc. for $\mathrm{C}_{10} \mathrm{H}_{9} \mathrm{FN}, 162.07135$ found 162.07112 .

2-(Fluoromethyl)-6-methylquinoline $(\mathbf{2 h})^{13}$

Procedure B; Brown solid, yield: $14.4 \mathrm{mg}(22 \%)$.

${ }^{1} \mathrm{H} \mathrm{NMR}\left(\mathrm{CDCl}_{3}, 300 \mathrm{MHz}\right): \delta=7.96(\mathrm{~d}, \mathrm{~J}=8.5 \mathrm{~Hz}, 1 \mathrm{H}), 7.88(\mathrm{~d}, \mathrm{~J}=8.5 \mathrm{~Hz}, 1 \mathrm{H}), 7.44(\mathrm{~m}, 3 \mathrm{H}), 5.56(\mathrm{~d}, \mathrm{~J}=$ $47.1 \mathrm{~Hz}, 2 \mathrm{H}), 2.42(\mathrm{~s}, 3 \mathrm{H})$.

${ }^{13} \mathrm{C} \mathrm{NMR}\left(\mathrm{CDCl}_{3}, 300 \mathrm{MHz}\right): \delta=155.6(\mathrm{~d}, \mathrm{~J}=21.5 \mathrm{~Hz}), 145.8,136.5,136.3,132.1,128.5,127.6,126.4$, $118.2(\mathrm{~d}, \mathrm{~J}=4.9 \mathrm{~Hz}), 85.0(\mathrm{~d}, \mathrm{~J}=169.8 \mathrm{~Hz}), 21.4$.

${ }^{19} \mathrm{~F} \mathrm{NMR}(\mathrm{CDCl} 3,282.2 \mathrm{MHz}): \delta=-220.30(\mathrm{td}, \mathrm{J}=47.0,3.3 \mathrm{~Hz})$.

HRMS: $\mathrm{m} / \mathrm{z}[\mathrm{M}+\mathrm{H}]+$ calc. for $\mathrm{C}_{11} \mathrm{H}_{11} \mathrm{FN}, 176.0870$ found 176.0871 .

3-(fluoromethyl)benzo[f]quinoline (2i) $)^{13}$

Procedure B; Brown solid, yield: $21.4 \mathrm{mg}(27 \%)$.

${ }^{1} \mathrm{H} \mathrm{NMR}\left(\mathrm{CDCl}_{3}, 300 \mathrm{MHz}\right): \delta=8.92(\mathrm{~d}, \mathrm{~J}=8.6 \mathrm{~Hz}, 1 \mathrm{H}), 8.58-8.49(\mathrm{~m}, 1 \mathrm{H}), 7.98-7.89(\mathrm{~m}, 3 \mathrm{H}), 7.72-$ $7.61(\mathrm{~m}, 3 \mathrm{H}), 5.69(\mathrm{~d}, \mathrm{~J}=47.0 \mathrm{~Hz}, 2 \mathrm{H})$.

${ }^{13} \mathrm{C} \mathrm{NMR}\left(\mathrm{CDCl}_{3}, 300 \mathrm{MHz}\right): \delta=156.2(\mathrm{~d}, \mathrm{~J}=21.9 \mathrm{~Hz}), 147.5(\mathrm{~d}, \mathrm{~J}=1.7 \mathrm{~Hz}), 131.8,131.7,131.5,129.5$, 128.8, 127.8, 127.5, 127.3, 124.9 (d, J = 0.9 Hz), 122.7, 118.4 (d, J = 5.4 Hz), 84.9 (d, J = $170.0 \mathrm{~Hz}$ ).

HRMS: $\mathrm{m} / \mathrm{z}[\mathrm{M}+\mathrm{H}]+$ calc. for $\mathrm{C}_{14} \mathrm{H}_{11} \mathrm{FN}, 212.0870$ found 212.0869 .

$\underline{\text { 6-Bromo-2-(Fluoromethyl)quinoline }(2 j)^{13}}$

Procedure B; Brown solid, yield: $16 \mathrm{mg}(18 \%)$.

${ }^{1} \mathrm{H} \mathrm{NMR}\left(\mathrm{CDCl}_{3}, 300 \mathrm{MHz}\right): \delta=8.10(\mathrm{~d}, \mathrm{~J}=8.5 \mathrm{~Hz}, 1 \mathrm{H}), 7.96(\mathrm{~d}, \mathrm{~J}=2.1 \mathrm{~Hz}, 1 \mathrm{H}), 7.89(\mathrm{~d}, \mathrm{~J}=9.0 \mathrm{~Hz}, 1 \mathrm{H})$, $7.76(\mathrm{dd}, \mathrm{J}=9.0,2.2 \mathrm{~Hz}, 1 \mathrm{H}), 7.59(\mathrm{dd}, \mathrm{J}=8.5,0.9 \mathrm{~Hz}, 1 \mathrm{H}), 5.61(\mathrm{~d}, \mathrm{~J}=46.9 \mathrm{~Hz}, 2 \mathrm{H})$.

${ }^{13} \mathrm{C} \mathrm{NMR}\left(\mathrm{CDCl}_{3}, 300 \mathrm{MHz}\right): \delta=157.4(\mathrm{~d}, \mathrm{~J}=21.9 \mathrm{~Hz}), 146.0,136.2,133.5,130.8,129.8,128.8,120.7$, $119.1(\mathrm{~d}, \mathrm{~J}=5.2 \mathrm{~Hz}), 84.9(\mathrm{~d}, \mathrm{~J}=170.8 \mathrm{~Hz})$.

${ }^{19} \mathrm{~F} \mathrm{NMR}\left(\mathrm{CDCl}_{3}, 282.2 \mathrm{MHz}\right): \delta=-221.94(\mathrm{t}, \mathrm{J}=47.0 \mathrm{~Hz})$.

HRMS: $\mathrm{m} / \mathrm{z}[\mathrm{M}+\mathrm{H}]+$ calc. for $\mathrm{C}_{10} \mathrm{H}_{8} \mathrm{FN}, 239.9819$ found 239.9818 .

6-Chloro-2-(Fluoromethyl)quinoline $(\mathbf{2 k})^{13}$

Procedure B; Yellow solid, yield: 14.6 mg (20\%). 
${ }^{1} \mathrm{H} \mathrm{NMR}\left(\mathrm{CDCl}_{3}, 300 \mathrm{MHz}\right): \delta=8.09(\mathrm{~d}, \mathrm{~J}=8.5 \mathrm{~Hz}, 1 \mathrm{H}), 7.95(\mathrm{~d}, \mathrm{~J}=9.0 \mathrm{~Hz}, 1 \mathrm{H}), 7.76(\mathrm{~d}, \mathrm{~J}=2.0 \mathrm{~Hz}, 1 \mathrm{H})$, $7.67-7.53(\mathrm{~m}, 2 \mathrm{H}), 5.61(\mathrm{~d}, \mathrm{~J}=46.9 \mathrm{~Hz}, 2 \mathrm{H})$.

${ }^{13} \mathrm{C} \mathrm{NMR}\left(\mathrm{CDCl}_{3}, 300 \mathrm{MHz}\right): \delta=157.2(\mathrm{~d}, \mathrm{~J}=21.9 \mathrm{~Hz}), 145.8,136.3,132.6,131.0,130.7,128.3,126.4$, $119.2(\mathrm{~d}, \mathrm{~J}=5.2 \mathrm{~Hz})$.

${ }^{19} \mathrm{~F} \mathrm{NMR}(\mathrm{CDCl} 3,282.2 \mathrm{MHz}): \delta=-221.81(\mathrm{t}, \mathrm{J}=46.9 \mathrm{~Hz})$.

HRMS: $\mathrm{m} / \mathrm{z}[\mathrm{M}+\mathrm{H}]+$ calc. for $\mathrm{C}_{10} \mathrm{H}_{8} \mathrm{ClFN}, 196.0324$ found 196.0322 .

6-Chloro-2-(Fluoromethyl)quinoline $(2 \mathrm{~m})^{13}$

Procedure B; Yellow solid, yield: $21.0 \mathrm{mg}(32 \%)$.

${ }^{1} \mathrm{H} \mathrm{NMR}\left(\mathrm{CDCl}_{3}, 300 \mathrm{MHz}\right): \delta=8.01-7.92(\mathrm{~m}, 1 \mathrm{H}), 7.87(\mathrm{dd}, \mathrm{J}=8.4,1.0 \mathrm{~Hz}, 1 \mathrm{H}), 7.60$ (ddd, J = 8.4, 6.9, $1.4 \mathrm{~Hz}, 1 \mathrm{H}$ ), 7.45 (ddd, J = 8.2, 6.9, $1.2 \mathrm{~Hz}, 1 \mathrm{H}), 7.31(\mathrm{~s}, 1 \mathrm{H}), 5.50(\mathrm{~d}, \mathrm{~J}=47.1 \mathrm{~Hz}, 2 \mathrm{H}), 2.61(\mathrm{~s}, 3 \mathrm{H})$.

${ }^{13} \mathrm{C} \mathrm{NMR}\left(\mathrm{CDCl}_{3}, 300 \mathrm{MHz}\right): \delta=156.49$ (d, J = 21.2 Hz), 147.3, 145.6, $129.6(2 \mathrm{C}), 127.7,126.6,123.9$, $119.0(\mathrm{~d}, \mathrm{~J}=4.9 \mathrm{~Hz}), 85.1(\mathrm{~d}, \mathrm{~J}=170.0 \mathrm{~Hz}), 18.9$.

${ }^{19} \mathrm{~F} \mathrm{NMR}\left(\mathrm{CDCl}_{3}, 282.2 \mathrm{MHz}\right): \delta=-221.22(\mathrm{t}, \mathrm{J}=46.9 \mathrm{~Hz})$.

HRMS: $\mathrm{m} / \mathrm{z}[\mathrm{M}+\mathrm{H}]+$ calc. for $\mathrm{C}_{10} \mathrm{H}_{8} \mathrm{ClFN}, \mathrm{C} 11 \mathrm{H} 11 \mathrm{FN}, 177.0948$ found 177.0950 .

4-Fluoro-1,2,3,4-tetrahydroacridine (2n)

Procedure B; Yellow oil, yield: $31.6 \mathrm{mg}(42 \%)$.

${ }^{1} \mathrm{H} \mathrm{NMR}\left(\mathrm{CDCl}_{3}, 300 \mathrm{MHz}\right): \delta=8.15(\mathrm{~d}, \mathrm{~J}=8.5 \mathrm{~Hz}, 1 \mathrm{H}), 7.95(\mathrm{~s}, 1 \mathrm{H}), 7.75(\mathrm{~d}, \mathrm{~J}=8.2 \mathrm{~Hz}, 1 \mathrm{H}), 7.67$ (ddd, J $=8.4,6.9,1.4 \mathrm{~Hz}, 1 \mathrm{H}), 7.52(\mathrm{dd}, \mathrm{J}=8.0,7.0 \mathrm{~Hz}, 1 \mathrm{H}), 5.76(\mathrm{dt}, \mathrm{J}=7.5,3.4 \mathrm{~Hz}, 1 \mathrm{H}), 3.09(\mathrm{dt}, \mathrm{J}=16.0,4.2$ $\mathrm{Hz}, 1 \mathrm{H}), 2.98-2.86(\mathrm{~m}, 1 \mathrm{H}), 2.61-2.35(\mathrm{~m}, 1 \mathrm{H}), 2.27-2.04(\mathrm{~m}, 2 \mathrm{H}), 1.91(\mathrm{dd}, \mathrm{J}=9.1,3.2 \mathrm{~Hz}, 1 \mathrm{H})$.

${ }^{13} \mathrm{C} \mathrm{NMR}\left(\mathrm{CDCl}_{3}, 300 \mathrm{MHz}\right): \delta=153,4(\mathrm{~d}, \mathrm{~J}=15.2 \mathrm{~Hz}), 146.6,136.3,130.8,129.2,129.1,128.4,128.3$ (d, J = 1.5 Hz), 127.1 (d, J = 21.4 Hz), 89.1 (d, J = 169.4 Hz), 29.9 (d, J = 21.3 Hz), 28.5, 17.6 (d, J = 2.2 $\mathrm{Hz})$.

${ }^{19} \mathrm{~F} \mathrm{NMR}\left(\mathrm{CDCl}_{3}, 282.2 \mathrm{MHz}\right): \delta=-162.92$ (ddd, J = 41.0, 29.9, $\left.15.2 \mathrm{~Hz}, 1 \mathrm{~F}\right)$.

HRMS: $\mathrm{m} / \mathrm{z}[\mathrm{M}+\mathrm{H}]+$ calc. for $\mathrm{C}_{13} \mathrm{H}_{13} \mathrm{FN}, 202.1027$ found 202.1023 .

6-Chloro-2-(Fluoromethyl)quinoline (20)

Procedure B; Yellow oil, yield: $28.2 \mathrm{mg}(32 \%)$.

${ }^{1} \mathrm{H} \mathrm{NMR}\left(\mathrm{CDCl}_{3}, 300 \mathrm{MHz}\right): \delta=8.22(\mathrm{~d}, \mathrm{~J}=8.4 \mathrm{~Hz}, 1 \mathrm{H}), 8.15(\mathrm{~d}, \mathrm{~J}=8.4 \mathrm{~Hz}, 1 \mathrm{H}), 7.80-7.69(\mathrm{~m}, 1 \mathrm{H}), 7.63$ (dd, J = 8.0, 7.2 Hz, 1H), $5.72(d t, J=7.5,3.5 \mathrm{~Hz}, 1 \mathrm{H}), 3.34-3.16(\mathrm{~m}, 1 \mathrm{H}), 2.87(\mathrm{dq}, \mathrm{J}=10.4,4.3 \mathrm{~Hz}$, $1 \mathrm{H}), 2.48$ (ddd, J = 19.7, 10.8, 4.4 Hz, 1H), $2.19-1.93(\mathrm{~m}, 3 \mathrm{H})$.

${ }^{13} \mathrm{C} \mathrm{NMR}\left(\mathrm{CDCl}_{3}, 300 \mathrm{MHz}\right): \delta=153,7(\mathrm{~d}, \mathrm{~J}=15.4 \mathrm{~Hz}), 147.1,142.1,129.8(\mathrm{~d}, \mathrm{~J}=7.8 \mathrm{~Hz}), 129.0,128.0$, $126.4,123.7,89.4(\mathrm{~d}, \mathrm{~J}=170.8 \mathrm{~Hz}), 29.2(\mathrm{~d}, \mathrm{~J}=21.4 \mathrm{~Hz}), 26.8,16.9(\mathrm{~d}, \mathrm{~J}=2.6 \mathrm{~Hz})$.

${ }^{19} \mathrm{~F} \mathrm{NMR}\left(\mathrm{CDCl}_{3}, 282.2 \mathrm{MHz}\right): \delta=-164.27--164.79(\mathrm{~m}, 1 \mathrm{~F})$.

HRMS: $\mathrm{m} / \mathrm{z}[\mathrm{M}+\mathrm{H}]+$ calc. for $\mathrm{C}_{13} \mathrm{H}_{12} \mathrm{ClFN}, 236.0637$ found 236.0636 .

\section{Funding Information}

This work was supported by the CNRS and Lille University (PEPS 174920).

\section{Acknowledgment}

The NMR and Mass Spectrometry facilities used in this study were funded by the European Community (FEDER), Région Haut de France (France), the CNRS, and Lille University.

\section{Supporting Information}

YES

\section{References}


(1) (a) Walsh, C. T. Tetrahedron Lett., 2015, 56, 3075. (b) Vitaku, E., Smith T. D.; Njardarson, J. T.; J. Med. Chem., 2014, 57, 10257. (c) Baumann, M.; Baxendale, I. R. ; Beilstein J. Org. Chem., 2013, 9, 2265.(d) J. P. Michael, Nat. Prod. Rep., 2005, 22, 627.

(2) (a) Hu, Y.-Q.; Gao, C.; Zhang, S.; Xu, L.; Xu, Z.; Feng, L.-S.; Wu, X.; Zhao, F.; Eur. J. Med. Chem., 2017, 139, 22; (b) Froehlich, T.; Tsogoeva, S. B.; J. Med. Chem., 2016, 59, 9668. (c) Golden, E. B.; Cho, H.-Y.; Hofman, F. M.; Louie, S. G.; Schonthal A. H.; Chen, T. C.; Neurosurg Focus, 2015, 38, E12. (d) Teguh, S. C.; Klonis, N.; Duffy, S.; Lucantoni, L.; Avery, V. M.; Hutton, C. A.; Baell J. B.; Tilley, L.; J. Med. Chem., 2013, 56, 6200.

(3) (a) Aly, R. M.; Serya, R. A. T.; El-Motwally, A. M.; Esmat, A.; Abbas S. ; Abou El Ella, D. A.; Bioorg. Chem., 2017, 75, 368; (b) Kukowska, M.; Eur. J. Pharm. Sci., 2017, 109, 587. (c) Afzal, O.; Kumar, S.; Haider, M. R.; Ali, M. R.; Kumar, R.; Jaggi M.; Bawa, S.; Eur. J. Med. Chem., 2015, 97, 871. (d) Harding, M. M.; Long G. V.; Brown, C. L.; J. Med. Chem., 1993, 36, 3056.

(4) (a) Ghattas, A.-E.-B. A. G.; Khodairy, A.; Moustafa, H. M.; Hussein, B. R. M.; Farghaly M. M.; Aboelez, M. O.; Pharm. Chem. J., 2017, 51, 652; (b) Hassan, H. M.; Boonlarppradab C., Fenical, W.; J. Antibiot., 2016, 69, 511.

(5) (a) Vacher, B.; Bonnaud, B.; Funes, P.; Jubault, N.; Koek, W.; Assie, M.-B.; Cosi C.; Kleven, M.; J. Med. Chem., 1999, 42, 1648.

(6) (a) Shaw, A. T.; Yeap, B. Y.; Solomon, B. J.; Riely, G. J.; Gainor, J.; Engelman, J. A.; Shapiro, G. I.; Costa, D. B.; Ou, S.-H. I.; Butaney, M.; Salgia, R.; Maki, R. G.; Varella-Garcia, M.; Doebele, R. C.; Bang, Y.-J.; Kulig, K. ; Selaru, P.; Tang, Y.; Wilner, K. D.; Kwak, E. L.; Clark, J. W.; lafrate A. J.; Camidge, D. R. Lancet Oncol., 2011, 12, 1004.

(7) Verhoest, P. R.; Chapin, D. S.; Corman, M.; Fonseca, K.; Harms, J. F. X.; Hou, Marr, E. S.; Menniti, F. S.; Nelson, F.; O'Connor, R.; Pandit, J.; Proulx-LaFrance, C.; Schmidt, A. W. ;Schmidt, C. J.; Suiciak J. A.; Liras, S.; J. Med. Chem., 2009, 52, 5188

(8) Rocher, J.-P.; Bonnet, B.; Bolea, C.; Lutjens, R.; Le Poul, E.; Poli, S.; Epping-Jordan, M.; Bessis, A.-S.; Ludwig, B.; Mutel, V.; Curr. Top. Med. Chem., 2011, 11, 680.

(9) Sameem, B.; Saeedi, M.; Mahdavi M.; Shafiee, A.; Eur. J. Med. Chem., 2016, 128, 332.

(10) Watkins, P. B.; Zimmerman, H. J.; Knapp, M. J.; Gracon S. I.; Lewis, K. W.; J. Am. Med. Assoc., 1994, 271, 992.

(11) (a) Meanwell, N. A.; J. Med. Chem., 2018, 61, 5822. (b) Gillis, E. P.; Eastman, K. J.; Hill, M. D.; Donnelly, D. J.; Meanwell, N. A.; J. Med. Chem., 2015, 58, 8315. (c) Meanwell, N. A.; J. Med. Chem., 2011, 54, 2529. (d) Purser, S.; Moore, P. R.; Swallow, S.; Gouverneur, V.; Chem. Soc. Rev., 2008, 37, 320; (e) Muller, K.; Faeh C.; Diederich, F., Science, 2007, 317, 1881.

(12) (a) Kaur, M.; Van Humbeck, J. F.; Org. Biomol. Chem., 2020, 18, 606. (b) Latha D. S.; Yaragorla, S.; Eur. J. Org., 2020, 2155.

(13) For selective exemples see: (a) Pang, X.; Xiang, L.; Ma, J.; Yang, X.; Yan, R.; RSC Advances, 2016, 6, 111713. (b) Schmeck, C.; Gielen-Haertwig, H.; Vakalopoulos, A.; Bischoff, H., Li, V.; Wirtz, G.; Weber, O.; Bioorg. Med. Chem. Lett., 2010, 20, 1740.

(14) Kawanishi, R.; Phongphane, L.; Iwasa, S.; Shibatomi, K.; Chem. Eur. J., 2019, 25, 7453. 
(15) (a) Szpera, R.; Moseley, D. F. J.; Smith, L. B.; Sterling, A. J.; Gouverneur, V.; Angew. Chem., Int. Ed., 2019, 58, 14824. (b) Bume, D. D.; Harry, S. A.; Lectka, T.; Pitts, C. R.; J. Org. Chem., 2018, 83, 8803. (c) Ishida, S.; Sheppard, T.; Nishikata, T.; Tetrahedron Lett., 2018, 59, 789.(d) Koperniku, A.; Liu, H.; Hurley, P. B.; Eur. J. Org. Chem., 2016, 871. (e) Champagne, P. A.; Desroches, J. ; Hamel, J.-D. ; Vandamme, M. ; Paquin, J.-F. ; Chem. Rev., 2015, 115, 9073.

(16) McMurtrey, K. B.; Racowski, J. M.; Sanford, M. S.; Org. Lett., 2012, 14, 4094.

(17) Ying, W.; DesMarteau, D. D.; Gotoh, Y.; Tetrahedron, 1996, 52, 15.

(18) (a) Meanwell, M.; Nodwell, M. B.; Martin, R. E.; Britton, R.; Angew. Chem., Int. Ed., 2016, 55, 13244; (b) Meanwell, M.; Adluri, B. S.; Yuan, Z.; Newton, J.; Prevost, P.; Nodwell, M. B.; Friesen, C. M.; Schaffer, P.; Martin, R. E.; Britton, R.; Chem. Sci., 2018, 9, 5608.

(19) Danahy, K. E.; Cooper, J. C.; Van Humbeck, J. F.; Angew. Chem., Int. Ed., 2018, 57, 5134. 